Research Article

\title{
Topp-Leone Fréchet Distribution with Theory and Application
}

\author{
Laxmi Prasad Sapkota \\ Lecturer, Tribhuvan University, Tribhuvan Multiple Campus, Palpa
}

Corresponding email: laxmisapkota75@gmail.com

Article History

\begin{abstract}
We have defined a new distribution as a generalization of Frechet distribution called the ToppLeone Frechet distribution. Some properties and characteristics of the proposed distribution are presented. The hazard rate function can have the shape of the reverse-j, right-skewed and unimodal. The parameters of the proposed model are estimated by employing the maximum likelihood estimation (MLE) method and asymptotic confidence intervals for MLEs are also provided. $R$ software is used for computational purpose. The application of the Topp-Leone Frechet distribution is presented by considering a real dataset and found that the proposed model provided a good fit as compared with some competing models.

Keywords: Estimation, Frechet distribution, moments, survival function, Topp-Leone generator

\section{INTRODUCTION}

In the literature of probability theory and inferences, it has been observed that the various lifetime distributions have been constructed but the real datasets associated with engineering, life sciences, hydrology, life testing, biology and risk analysis do not produce a good fit to these distributions. So, the modification of the existing distributions appears to be essential to deal with the weaknesses in these fields. The generalized, extended, transmuted and modified distributions are developed by adding one or more parameters or performing some transformation to the parent distribution consequently, the newly generated distributions can provide a better fit as compared to the existing distributions.
\end{abstract}

(C) The Author, published by JRCC, Janapriya Multiple Campus. 
In this study, we have taken the one parameter Fréchet model which is the important model in extreme value theory and it can be applied in various fields like accelerated life testing, floods, earthquakes, horse racing, queues in supermarkets, rainfall, sea waves and wind speeds, etc. The cumulative distribution function (CDF) and probability density function (PDF) of one parameter Fréchet distribution respectively can be written as

$$
\begin{aligned}
& G(x ; \lambda)=\exp \left(-x^{-\lambda}\right) ; x>0, \lambda>0 \text { and } \ldots . . . \\
& g(x ; \lambda)=\lambda x^{-(1+\lambda)} \exp \left(-x^{-\lambda}\right) ; x>0, \lambda>0 .
\end{aligned}
$$

The generalization of the Frechet distribution is developed and studied by different researchers as detailed information regarding the Frechet distribution and its applications, readers can go through ((Kotz \& Nadarajah, 2000), (Harlow, 2002)). da Silva et al., (2013) have been studied the gamma extended Fréchet distribution. Mahmoud and Mandouh (2013) introduced the transmuted Fréchet distribution. Similarly, another extension of Frechet distribution was presented by (Krishna et al., 2013) using Marshall-Olkin-G family of distribution. The transmuted Marshall-Olkin Fréchet distribution was introduced by (Afify et al., 2014), The Weibull Fréchet distribution was generated by (Afify et al., 2016). Mead et al., (2017) has been defined the beta exponential Fréchet distribution, Korkmaz et al., (2017) introduced the odd Lindley Fréchet distribution, Yousof and Hamedani (2018) have presented another extension of Frechet distribution with regression models. Further, the Gompertz fréchet distribution was defined by (Oguntunde et al., 2019), and the compound Frechet distribution was defined by (Yousof et al., 2019). Hence in this study, we are interested in the modification of Frechet distribution by using the Topp-Leone-G family defined by (Al-Shomrani et al., 2016). The cumulative distribution function (CDF) and probability density function of the Topp-Leone generating family can be expressed as

$$
\begin{aligned}
& F(x)=\left[1-\{1-G(x)\}^{2}\right]^{\alpha} ; x \in \mathfrak{R}, \alpha>0 \text { and ........... (3) } \\
& f(x)=2 \alpha g(x)\{1-G(x)\}\left[1-\{1-G(x)\}^{2}\right]^{\alpha-1} ; x \in \mathfrak{R}, \alpha>0
\end{aligned}
$$

respectively. Where $G(x) g(x)$ and are $\mathrm{CDF}$ and PDF of any parent distribution and $\alpha$ is the shape parameter of G-family. This generating family has also been used by 
(Abbas et al., 2017) as Topp-Leone inverse Weibull distribution and the Topp-Leone Lomax distribution by (Oguntunde et al., 2019).

The main objective of this work is to develop a more flexible lifetime distribution by inserting just one extra parameter to one parameter Fréchet distribution to obtain a better fit to the real data sets. The different sections of this article have been structured as follows; new distribution is defined in Section 2 and some important functional form of the proposed distribution is presented. In Section 3 We present some statistical and mathematical properties of Topp-Leone Frechet distribution. The parameters of the distribution are estimated using the maximum likelihood estimation (MLE) method and presented in Section 4. The application of the proposed distribution is illustrated using a real dataset in Section 5. The concluding remark of this work is presented in Section 6.

\section{The Topp-Leone Frechet (TLF) Distribution}

In this section, we have defined a new distribution using the Topp-Leone generating family of distribution to extend the one-parameter Frechet distribution called Topp-Leone Frechet distribution. The CDF and PDF of the TLF distribution are obtained by using (1) and (2) in (3) and (4). Let $\mathrm{X}$ be a non-negative random variable that follows TLF distribution if its CDF can take the following form

$$
F(x)=\left[1-\left\{1-\exp \left(-x^{-\lambda}\right)\right\}^{2}\right]^{\alpha} ; x>0, \alpha>0, \lambda>0
$$

and the corresponding PDF of (5) is

$$
f(x)=2 \alpha \lambda x^{-(1+\lambda)} \exp \left(-x^{-\lambda}\right)\left\{1-\exp \left(-x^{-\lambda}\right)\right\}\left[1-\left\{1-\exp \left(-x^{-\lambda}\right)\right\}^{2}\right]^{\alpha-1} ; x>0, \alpha>0, \lambda>0
$$

\section{The Reliability/Survival Function}

$$
\begin{aligned}
& R(x)=1-F(x) \\
& =1-\left[1-\left\{1-\exp \left(-x^{-\lambda}\right)\right\}^{2}\right]^{\alpha}
\end{aligned}
$$

Hazard Rate Function of TLF Distribution

The hazard rate function (HRF) can be expressed as, 


$$
\begin{aligned}
& h(x)=\frac{f(x)}{R(x)} \\
& =2 \alpha \lambda x^{-(1+\lambda)} \exp \left(-x^{-\lambda}\right) \frac{\left\{1-\exp \left(-x^{-\lambda}\right)\right\}}{1-\left[1-\left\{1-\exp \left(-x^{-\lambda}\right)\right\}^{2}\right]^{\alpha}}\left[1-\left\{1-\exp \left(-x^{-\lambda}\right)\right\}^{2}\right]^{\alpha-1}
\end{aligned}
$$

The shape of the PDF and HRF of TLF distribution are displayed in Figure 1. It is observed that the PDF of TLF distribution can have different varieties of shapes. The HRF for the TLF distribution is also exhibited flexibility due to its different shapes such as increasing, increasing-decreasing, reverse-J and upside-down bathtub for different values of parameters.

\section{Figure 1}

Graph of PDF (left panel) and HRf (right panel) for different values of $\alpha$ and $\lambda$.
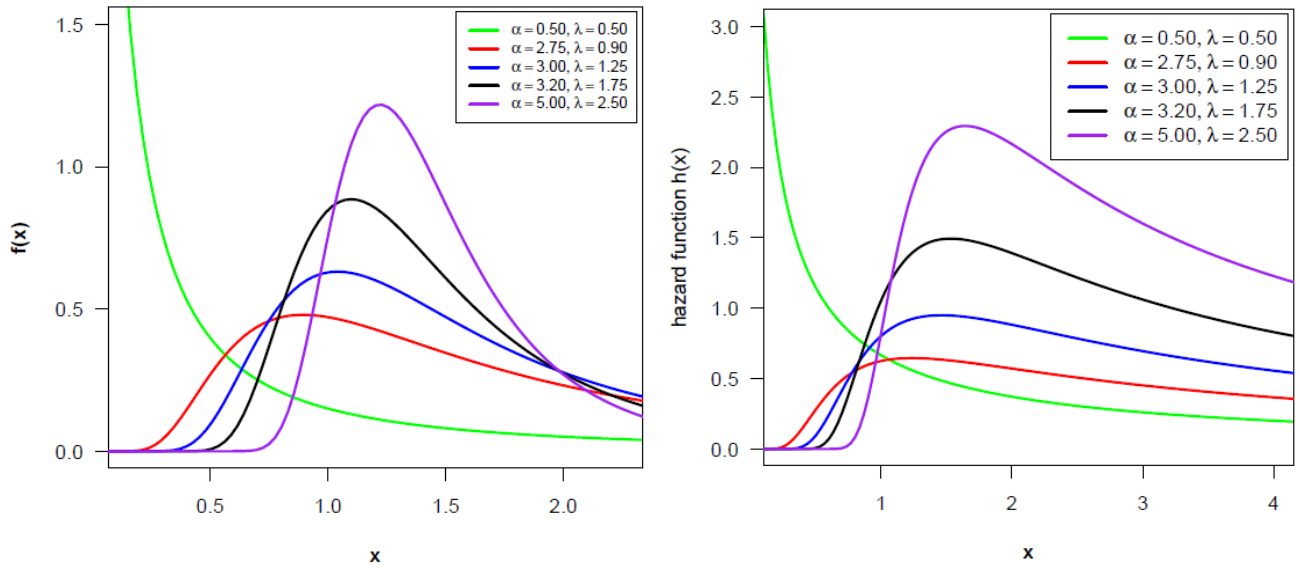

\section{Cumulative Hazard Function (CHF)}

The CHF of TLF distribution can be defined as

$$
\begin{aligned}
H(x)=\int_{-\infty}^{x} h(t) d t & \\
& =-\ln [1-F(x)] \\
& =-\ln \left[1-\left[1-\left\{1-\exp \left(-x^{-\lambda}\right)\right\}^{2}\right]^{\alpha}\right]
\end{aligned}
$$

Quantile Function of TLF Distribution 
The quantile function is defined as the inverse of the cumulative distribution function.

$$
Q(p)=F^{-1}(p)
$$

Hence quantile function of TLF distribution can be expressed as

$$
Q(p)=\left[-\log \left\{1-\sqrt{\left(1-p^{1 / \alpha}\right)}\right\}\right]^{-1 / \lambda} ; 0<p<1
$$

Generation of random deviation from the $\operatorname{TLF}(\alpha, \lambda)$ can be done using the following relation

$$
x=\left[-\log \left\{1-\sqrt{\left(1-v^{1 / \alpha}\right)}\right\}\right]^{-1 / \lambda} ; 0<v<1 .
$$

where $\mathrm{v}$ has the $U(0,1)$ distribution.

\section{Skewness and Kurtosis:}

The coefficient of skewness defined by Bowley's can be calculated as

$$
S_{k}(B)=\frac{Q(1 / 4)-2 Q(1 / 2)+Q(3 / 4)}{Q(3 / 4)-Q(1 / 4)} \text { and }
$$

The coefficient of kurtosis based on octiles defined by (Moors, 1988) is

$$
K(\text { Moors })=\frac{Q(0.875)+Q(0.375)-Q(0.625)-Q(0.125)}{Q(0.75)-Q(0.25)}
$$

\section{Statistical Properties of TLF Distribution}

\section{Useful Expansions of PDF and CDF of TLF Distribution}

For the series expansion of the PDF and CDF of TLF distribution we can use the generalized binomial expansion as

$$
(1-x)^{k}=\sum_{i=0}^{\infty}(-1)^{i}\left(\begin{array}{l}
k \\
i
\end{array}\right) x^{i} \text { for } k>0
$$

By using (12) we can expressed the PDF (6) of TLF distribution as

$$
f(x)=2 \alpha \lambda \sum_{i=0}^{\infty} \sum_{j=0}^{\infty} Z_{i j} x^{-(1+\lambda)} e^{-(1+j) x^{-\lambda}}
$$


where

$$
Z_{i j}=(-1)^{i+j}\left(\begin{array}{c}
\alpha-1 \\
i
\end{array}\right)\left(\begin{array}{c}
2 i+1 \\
j
\end{array}\right)
$$

Similarly the CDF (5) can be expressed as

$$
\begin{aligned}
{[F(x)]^{d} } & =\sum_{k=0}^{\infty} \sum_{l=0}^{\infty} \phi_{k l} e^{-l x^{-\lambda}} \\
\text { where } & \phi_{k l}=(-1)^{k+l}\left(\begin{array}{c}
\alpha d \\
k
\end{array}\right)\left(\begin{array}{c}
2 k \\
l
\end{array}\right)
\end{aligned}
$$

\section{Moments}

Let $\mathrm{X}$ be a non-negative random variable with PDF (13), then the $\mathrm{r}^{\text {th }}$ moments of TLF distribution can be defined as

$$
\begin{aligned}
& \mu_{r}^{\prime}=E\left(X^{r}\right)=\int_{0}^{\infty} x^{r} f(x) d x ; \mathrm{r}=1,2, \ldots \\
& =2 \alpha \lambda \sum_{i=0}^{\infty} \sum_{j=0}^{\infty} Z_{i j} \int_{0}^{\infty} x^{-(1+\lambda)+r} e^{-(1+j) x^{-\lambda}} d x
\end{aligned}
$$

let $x^{-\lambda}=t$ this gives $x=t^{-1 / \lambda}$ therefore $d x=(-1 / \lambda) t^{-\frac{1}{\lambda}-1}$

$$
\mu_{r}^{\prime}=-2 \alpha \sum_{i=0}^{\infty} \sum_{j=0}^{\infty} Z_{i j} \frac{\sqrt{\frac{\lambda-r}{\lambda}}}{(1+j)^{\frac{\lambda-r}{\lambda}}} \text { for } \lambda>r
$$

where $\Gamma(a)=\int_{0}^{\infty} x^{a-1} e^{-x} d x$ is the gamma function.

\section{Moment Generating Function (MGF)}

The MGF of the TLF distribution can be calculated using (15) as

$$
\begin{aligned}
& M_{X}(t)=E\left[e^{t x}\right]=\sum_{r=0}^{\infty} \frac{t^{r}}{r !} E\left(X^{r}\right)=-2 \alpha \sum_{i=0}^{\infty} \sum_{j=0}^{\infty} \sum_{r=0}^{\infty} \eta_{i j r} \frac{\sqrt{\frac{\lambda-r}{\lambda}}}{(1+j)^{\frac{\lambda-r}{\lambda}}}, \\
& \text { where } \eta_{i j r}=(-1)^{i+j}\left(\begin{array}{c}
\alpha-1 \\
i
\end{array}\right)\left(\begin{array}{c}
2 i+1 \\
j
\end{array}\right) \frac{t^{r}}{r !} .
\end{aligned}
$$


Probability Weighted Moments (PWM)

The PWM of the TLF distribution can be derived as

$$
M_{r, d}(X)=E\left[X^{r} F^{d}(x)\right]=\int_{0}^{\infty} x^{r} f(x) F^{d}(x) d x
$$

Using the (13) and (14) in (17) we can write

$$
\begin{aligned}
& M_{r, d}(X)=-2 \alpha \sum_{i=0}^{\infty} \sum_{j=0}^{\infty} \sum_{k=0}^{\infty} \sum_{l=0}^{\infty} Z_{i j k l}^{*} \frac{\sqrt{\frac{\lambda-r}{\lambda}}}{(1+j+l)^{\frac{\lambda-r}{\lambda}}}, \\
& \text { where } Z_{i j k l}^{*}=Z_{i j} \times \phi_{k l} \text { and } Z_{i j}=(-1)^{i+j}\left(\begin{array}{c}
\alpha-1 \\
i
\end{array}\right)\left(\begin{array}{c}
2 i+1 \\
j
\end{array}\right) \phi_{k l}=(-1)^{k+l}\left(\begin{array}{c}
\alpha d \\
k
\end{array}\right)\left(\begin{array}{c}
2 k \\
l
\end{array}\right) .
\end{aligned}
$$

\section{Entropy}

For the measurement of the variability of uncertainty in various fields like life science, physics, engineering, etc. we can apply the entropy measure. There are various types of entropies but we present here Renyi and q-entropy.

Renyi Entropy: The Renyi entropy of TLF distribution with PDF can be defined as

$$
I_{w}(X)=\frac{1}{1-w} \log \int_{0}^{\infty}\{f(x)\}^{w} d x, w>0 \text { and } w \neq 1 .
$$

Using (12) in PDF (6), then the PDF $\{f(x)\}^{w}$ can be written as

$$
\{f(x)\}^{w}=(2 \alpha \lambda)^{w} \sum_{i=0}^{\infty} \sum_{j=0}^{\infty} Z_{i j}^{*} x^{-(1+\lambda) w} e^{-(w+j) x^{-\lambda}}
$$

Hence

$$
\begin{aligned}
& Z_{i j}^{*}=(-1)^{i+j}\left(\begin{array}{c}
w(\alpha-1) \\
i
\end{array}\right)\left(\begin{array}{c}
2 i+w \\
j
\end{array}\right) . \\
& \text { where } \\
& I_{w}(X)=\frac{1}{1-w} \log \left[(2 \alpha \lambda)^{w} \sum_{i=0}^{\infty} \sum_{j=0}^{\infty} Z_{i j}^{*} \int_{0}^{\infty} x^{-(1+\lambda) w} e^{-(w+j) x^{-\lambda}} d x\right], w>0 \text { and } w \neq 1 .
\end{aligned}
$$

let $x^{-\lambda}=t$ this gives $x=t^{-1 / \lambda}$, therefore $d x=(-1 / \lambda) t^{-\frac{1}{\lambda}-1}$, then we can express the Renyi entropy of TLF distribution as 


$$
I_{w}(X)=\frac{1}{1-w} \log \left[-\lambda(2 \alpha \lambda)^{w} \sum_{i=0}^{\infty} \sum_{j=0}^{\infty} Z_{i j}^{*} \frac{\sqrt{1+(1+\lambda) \frac{w}{\lambda}}}{(w+j)^{1+(1+\lambda) \frac{w}{\lambda}}}\right] .
$$

Q-entropy: The q-entropy of the random variable X from TLF distribution can be defined as

$$
D_{q}(X)=\frac{1}{1-q} \log \left[1-\int_{0}^{\infty}\{f(x)\}^{q} d x\right], q \neq 1 \text { and } q>0 .
$$

Using (19) where w is replaced by q in (20) we get

$$
D_{q}(X)=\frac{1}{1-q} \log \left[1+\lambda(2 \alpha \lambda)^{w} \sum_{i=0}^{\infty} \sum_{j=0}^{\infty} Z_{i j}^{*} \frac{1+(1+\lambda) \frac{w}{\lambda}}{(w+j)^{1+(1+\lambda) \frac{w}{\lambda}}}\right], q \neq 1 \text { and } q>0 \text {. }
$$

\section{Order Statistics}

The $\mathrm{m}^{\text {th }}$ order statistic (David, 1981) can be calculated as

$$
\begin{aligned}
f_{m: n}(x) & =\frac{n !}{(m-1) !(n-m) !} f(x)[F(x)]^{m-1}[1-F(x)]^{m-r} \\
& =\frac{n !}{(m-1) !(n-m) !} f(x) \sum_{p=1}^{n-m}\left(\begin{array}{c}
n-m \\
p
\end{array}\right)[F(x)]^{p+m-1}
\end{aligned}
$$

Substituting PDF and CDF defined in (13) and (14) in (21) by replacing $\mathrm{d}$ by $\mathrm{p}+\mathrm{m}-1$ we get

$$
\begin{aligned}
& f_{m: n}(x)=\sum_{i=0}^{\infty} \sum_{j=0}^{\infty} \sum_{k=0}^{\infty} \sum_{l=0}^{\infty} \sum_{p=0}^{n-m} \Phi^{\prime}{ }_{i j k l p} \int_{0}^{\infty} x^{-(1+\lambda)+r} e^{-(1+j+l) x^{-\lambda}} d x \\
& \Phi_{i j k l p}=\frac{(2 \alpha \lambda) n !}{(m-1) !(n-m) !}(-1)^{i+j+k+l}\left(\begin{array}{c}
\alpha-1 \\
i
\end{array}\right)\left(\begin{array}{c}
2 i+1 \\
j
\end{array}\right)\left(\begin{array}{c}
\alpha(m+p-1) \\
k
\end{array}\right)\left(\begin{array}{c}
2 k \\
l
\end{array}\right) .
\end{aligned}
$$

Now the moments of the order statistics can be calculated as

$$
E\left(X_{m: n}^{r}\right)=\int_{0}^{\infty} x^{r} f_{m: n}(x) d x ; \mathrm{r}=1,2, \ldots,
$$




$$
\begin{aligned}
& =\sum_{i=0}^{\infty} \sum_{j=0}^{\infty} \sum_{k=0}^{\infty} \sum_{l=0}^{\infty} \sum_{p=0}^{n-m} \Phi^{\prime}{ }_{i j k l p} \int_{0}^{\infty} x^{-(1+\lambda)+r} e^{-(1+j+l) x^{-\lambda}} d x \\
& =\sum_{i=0}^{\infty} \sum_{j=0}^{\infty} \sum_{k=0}^{\infty} \sum_{l=0}^{\infty} \sum_{p=0}^{n-m} \Phi^{\prime}{ }_{i j k l p} \frac{\sqrt{\frac{1}{\lambda}(1-r+2 \lambda)}}{(1+j+l)^{\frac{1}{\lambda}(1-r+2 \lambda)}} \\
& \qquad \Phi^{\prime}{ }_{i j k l p}=\frac{-(2 \alpha) \times n !}{(m-1) !(n-m) !}(-1)^{i+j+k+l}\left(\begin{array}{c}
\alpha-1 \\
i
\end{array}\right)\left(\begin{array}{c}
2 i+1 \\
j
\end{array}\right)\left(\begin{array}{c}
\alpha(m+p-1) \\
k
\end{array}\right)\left(\begin{array}{c}
2 k \\
l
\end{array}\right) .
\end{aligned}
$$

\section{Maximum Likelihood Estimation}

In this section, we have introduced the maximum likelihood estimation (MLE) method to estimate the parameters of the TLF distribution. Let $\underline{x}=\left(x_{1}, \ldots, x_{n}\right)$ be a non-negative random sample of size ' $\mathrm{n}$ ' from $\operatorname{TLF}(\alpha, \lambda)$ then the likelihood function for $(\alpha, \lambda)$ is given by

$$
L(\alpha, \lambda)=\prod_{i=1}^{n}\left\{2 \alpha \lambda x_{i}^{-(1+\lambda)} \exp \left(-x_{i}^{-\lambda}\right)\left\{1-\exp \left(-x_{i}^{-\lambda}\right)\right\}\left[1-\left\{1-\exp \left(-x_{i}^{-\lambda}\right)\right\}^{2}\right]^{\alpha-1}\right\}
$$

Taking logarithm on both sides of (22) we get log-likelihood function as

$$
\log L=n \log (2 \alpha \lambda)-(1+\lambda) \sum_{i=1}^{n} x_{i}+\sum_{i=1}^{n} \log \left[1-\exp \left(-x_{i}^{-\lambda}\right)\right]+(\alpha-1) \sum_{i=1}^{n} \log \left[1-\left\{1-\exp \left(-x_{i}^{-\lambda}\right)\right\}\right]
$$

To maximize the log-likelihood function (23) directly we can use R software, Mathematica, Mat-lab, etc. or we can solve the likelihood equations which are obtained by differentiating (23) w. r. to the model parameters $\alpha$ and $\lambda$ as

$$
\begin{aligned}
& \frac{\partial \log L}{\partial \alpha}=\frac{n}{\alpha}+\sum_{i=1}^{n} \log \left[1-\left\{1-\exp \left(-x_{i}^{-\lambda}\right)\right\}\right] \\
& \frac{\partial \log L}{\partial \lambda}=\frac{n}{\lambda}-\sum_{i=1}^{n} x_{i}+(\alpha-2) \sum_{i=1}^{n} \frac{x_{i}^{-\lambda} \exp \left(-x_{i}^{-\lambda}\right) \log \left(x_{i}\right)}{1-\left\{1-\exp \left(-x_{i}^{-\lambda}\right)\right\}}
\end{aligned}
$$

Let $\hat{\alpha}$ and $\hat{\lambda}$ be the MLEs of $(\alpha, \lambda)$ which are the solutions of $\frac{\partial \log L}{\partial \alpha}=0 \frac{\partial \log L}{\partial \lambda}=0$ and for the construction of confidence intervals of the MLEs, 
we need to calculate the observed information matrix for this, first, we have to find the Fisher's information matrix given by,

$$
F=-\left(\begin{array}{cc}
E\left(\frac{\partial^{2} \log L}{\partial \alpha^{2}}\right) & E\left(\frac{\partial^{2} \log L}{\partial \alpha \partial \lambda}\right) \\
E\left(\frac{\partial^{2} \log L}{\partial \lambda \partial \alpha}\right) & E\left(\frac{\partial^{2} \log L}{\partial \lambda^{2}}\right)
\end{array}\right) .
$$

Again differentiating (24) and (25) w.r. to $\alpha$, and $\lambda$ we get $\frac{\partial^{2} \log L}{\partial \alpha^{2}}=-\frac{n}{\alpha^{2}}$ $\frac{\partial^{2} \log L}{\partial \lambda^{2}}=-\frac{n}{\lambda^{2}}+(\alpha-2) \sum_{i=1}^{n} \frac{x_{i}^{-\lambda} \exp \left(-x_{i}^{-\lambda}\right)\left[\log \left(x_{i}\right)\right]^{2}\left\{\left[1-\exp \left(-x_{i}^{-\lambda}\right)\right]\left[-x_{i}^{-\lambda}+1\right]-x_{i}^{-\lambda} \exp \left(-x_{i}^{-\lambda}\right)\right\}}{\left[1-\exp \left(-x_{i}^{-\lambda}\right)\right]^{2}}$

$$
\begin{aligned}
& \frac{\partial^{2} \log L}{\partial \alpha \partial \lambda}=\sum_{i=1}^{n} \frac{x_{i}^{-\lambda} \exp \left(-x_{i}^{-\lambda}\right) \log \left(x_{i}\right)}{1-\left\{1-\exp \left(-x_{i}^{-\lambda}\right)\right\}} \\
& \frac{\partial^{2} \log L}{\partial \lambda \partial \alpha}=\sum_{i=1}^{n} \frac{x_{i}^{-\lambda} \exp \left(-x_{i}^{-\lambda}\right) \log \left(x_{i}\right)}{1-\left\{1-\exp \left(-x_{i}^{-\lambda}\right)\right\}}
\end{aligned}
$$

In practice, we don't know $(\alpha, \lambda)$ hence we approximate the asymptotic variance by substituting the estimated value of the parameters $(\hat{\alpha}, \hat{\lambda})$. The observed information matrix ' $\mathrm{O}$ ' is calculated as

$$
O=-\left(\begin{array}{ll}
\frac{\partial^{2} \log L}{\partial \alpha^{2}} & \frac{\partial^{2} \log L}{\partial \alpha \partial \lambda} \\
\frac{\partial^{2} \log L}{\partial \lambda \partial \alpha} & \frac{\partial^{2} \log L}{\partial \lambda^{2}}
\end{array}\right)_{(\hat{\alpha}, \hat{\lambda})} .
$$

The observed information matrix is calculated by maximizing the likelihood using the Newton-Raphson algorithm. Therefore, the variance-covariance matrix is given by,

$$
[O]^{-1}=\left(\begin{array}{cc}
\operatorname{var}(\hat{\alpha}) & \operatorname{cov}(\hat{\alpha}, \hat{\lambda}) \\
\operatorname{cov}(\hat{\alpha}, \hat{\lambda}) & \operatorname{var}(\hat{\lambda})
\end{array}\right) .
$$

Hence confidence intervals for $\alpha$, and $\lambda$ at $100(1-\rho) \%$ can be created as, 


$$
\hat{\alpha} \pm Z_{\rho / 2} \sqrt{\operatorname{var}(\hat{\alpha})} \text {, and } \hat{\lambda} \pm Z_{\rho / 2} \sqrt{\operatorname{var}(\hat{\lambda})} \text {, }
$$

(24)

where $Z_{\rho / 2}$ denote the upper percentile of standard normal variate.

\section{Application with a Real Dataset}

For the illustration, we have taken a real dataset that was reported by (Hinkley, 1977). The data consists of the amount of precipitation (inches) having 30 successive values in March at Minneapolis/St Paul.

$2.05,2.10,3.00,1.95,0.90,0.81,2.81,0.59,1.43,1.20,0.52,3.09,1.74$, $0.32,1.51,1.87,0.47,1.89,2.20,1.62,3.37,1.35,0.77,2.48,4.75,0.96,1.31,1.20$, $1.18,0.81$

We have calculated the maximum likelihood estimator of TLF distribution directly by maximizing the likelihood function (23) using the optim(. ) function (Schmuller, 2017) in R software (R Core Team, 2020) and the Log-Likelihood value is $l=-40.59897$. In Table 1 we have displayed the MLE's and their corresponding standard errors (SE) and 95\% confidence intervals.

\section{Table 1}

MLE and SE and 95\% Confidence Interval for $\alpha$ and $\lambda$

\begin{tabular}{llll}
\hline Parameter & MLE & SE & $95 \%$ ACI \\
\hline alpha & 2.1104 & 0.3993 & $(1.3278,2.8930)$ \\
lambda & 1.0545 & 0.1414 & $(0.7774,1.3316)$ \\
\hline
\end{tabular}

Also, the variance-covariance matrix is

$$
\operatorname{var}-\operatorname{cov}=\left[\begin{array}{cc}
0.1594 & -0.0147 \\
-0.0147 & 0.0200
\end{array}\right]
$$

In Figure 2, we have displayed the Kolmogorov-Simnorov plot which shows that the TLF distribution can fits the data very well. 


\section{Figure 2}

Kolmogorov-Simnorov Plot of Fitted CDF with Empirical Distribution Function.

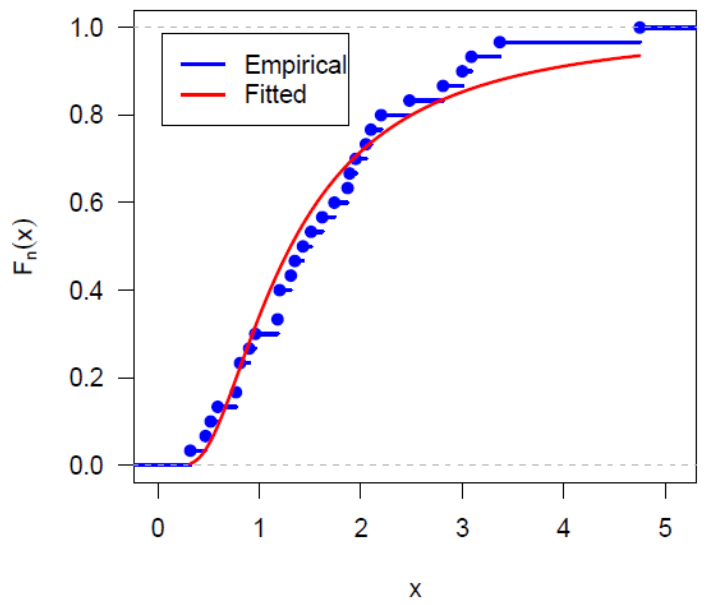

In Figure 3 we have plotted the Q-Q plot and P-P plot and it is observed that the proposed distribution fits the data very well.

\section{Figure 3}

The P-P Plot (left panel) and Q-Q Plot (right panel) of the TLF Distribution.
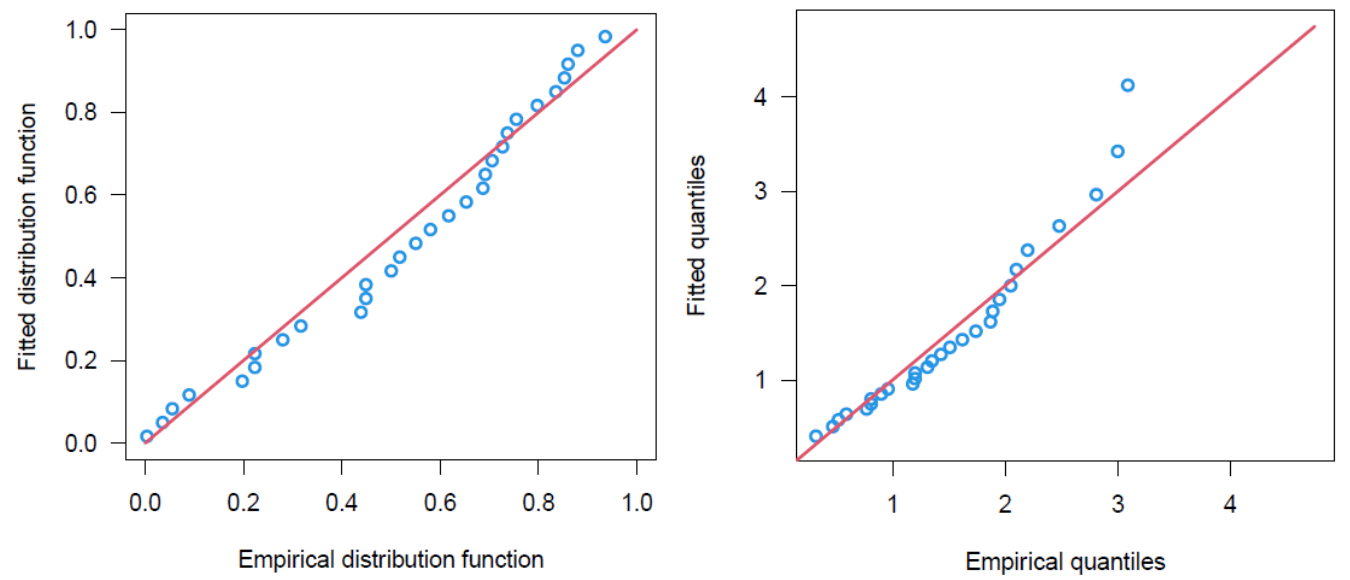

To demonstrate the capability of fit of the TLF distribution, some competing distributions are selected and fitted to compare the fit attain by TLF distribution, which are as follows,

A. Frechet Distribution (Fre2)

The PDF of two-parameter Frechet distribution is 


$$
f(x)=\beta \alpha^{\beta} x^{-\beta-1} \exp \left[-(\alpha / x)^{\beta}\right] ; x>0
$$

B. Exponential Power (EP) Distribution

The PDF of Exponential power (EP) distribution (Smith \& Bain, 1975) is

$$
f_{E P}(x)=\alpha \lambda^{\alpha} x^{\alpha-1} e^{(\lambda x)^{\alpha}} \exp \left\{1-e^{(\lambda x)^{\alpha}}\right\} ;(\alpha, \lambda)>0, \quad x \geq 0 .
$$

where $\alpha$ and $\lambda$ are the shape and scale parameters, respectively.

\section{Gompertz Distribution (GZ)}

The PDF of Gompertz distribution (Murthy et al., 2003) with parameters $\alpha$ and $\theta$ is

$$
f_{G Z}(x)=\theta e^{\alpha x} \exp \left\{\frac{\theta}{\alpha}\left(1-e^{\alpha x}\right)\right\} ; x \geq 0, \theta>0,-\infty<\alpha<\infty .
$$

D. Lindley Half Cauchy Distribution (LHC)

The PDF of LHC distribution (Chaudhary \& Kumar, 2020) with parameters $\theta$ and $\lambda$ is

$$
f(x)=\frac{2}{\pi}\left(\frac{\theta^{2}}{1+\theta}\right)\left(\frac{\lambda}{\lambda^{2}+x^{2}}\right)\left\{1-\frac{2}{\pi} \tan ^{-1}\left(\frac{x}{\lambda}\right)\right\}^{\theta-1}\left\{1-\ln \left[1-\frac{2}{\pi} \tan ^{-1}\left(\frac{x}{\lambda}\right)\right]\right\} ; \theta, \lambda>0, x>0
$$

For the assessment of potentiality of the proposed model we have calculated the log-likelihood, Akaike information criterion (AIC), Corrected Akaike information criterion (CAIC), Bayesian information criterion (BIC), and HannanQuinn information criterion (HQIC) which are presented in Table 2.

\section{Table 2}

Log-Likelihood (LL), AIC, BIC, AICC and HQIC

\begin{tabular}{llllll}
\hline Distribution & -LL & AIC & BIC & AICC & HQIC \\
\hline TLF & 40.5990 & 85.1979 & 88.0003 & 85.6424 & 86.0944 \\
FRE2 & 41.9170 & 87.8340 & 90.6364 & 88.2785 & 88.7305 \\
EP & 40.4769 & 84.9537 & 87.7561 & 85.3982 & 85.8503 \\
GZ & 41.0762 & 86.1523 & 88.9547 & 86.5968 & 87.0488 \\
LHC & 44.5938 & 93.1877 & 95.9900 & 93.6321 & 94.0842 \\
\hline
\end{tabular}


We have depicted the Histogram and the PDF of the fitted distributions and the Empirical distribution functions with the estimated distribution function of TLF distribution and some selected distributions in Figure 5.

\section{Figure 5}

Histogram and PDF of Fitted Distributions (left panel) and Empirical Vs Estimated Distribution Function (right panel)
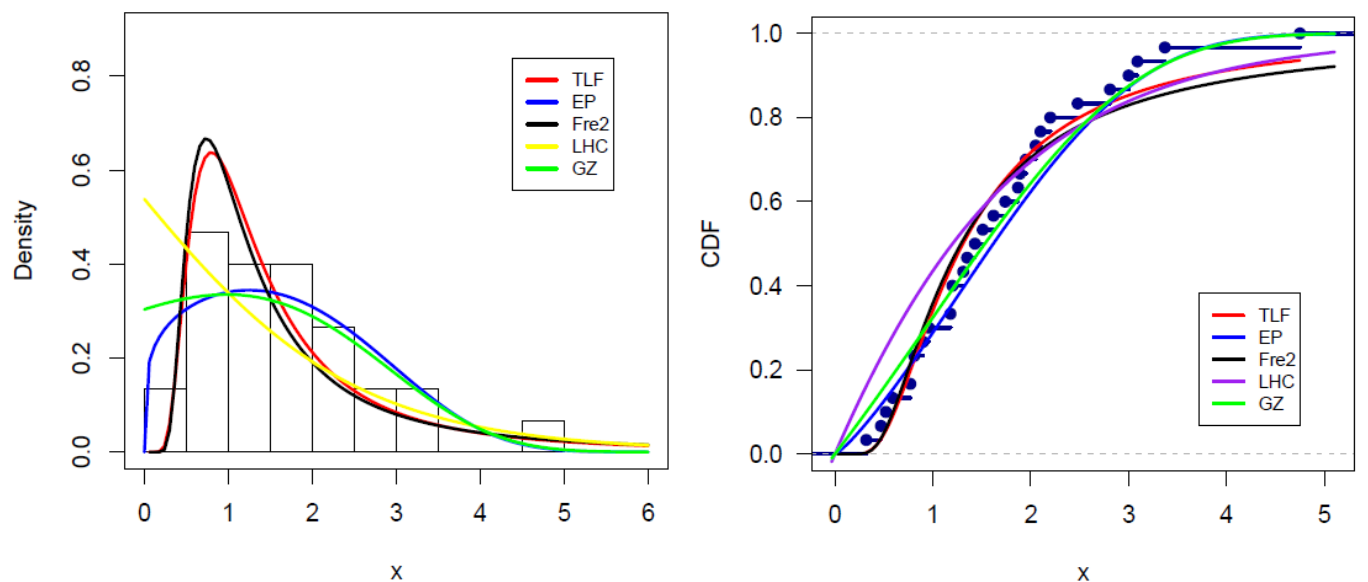

To compare the goodness-of-fit of the TLF distribution with other competing distributions we have presented the value of Kolmogorov-Simnorov (KS), the Anderson-Darling (W) and the Cramer-Von Mises $\left(\mathrm{A}^{2}\right)$ statistic with their corresponding $\mathrm{p}$-values in Table 3 . It is observed that the TLF distribution has the minimum value of the test statistic and a higher $p$-value thus we conclude that the TLF distribution gets quite a better fit and more consistent and reliable results than others taken for comparison.

\section{Table 3}

Goodness-of-Fit Statistics and Their Corresponding p-value

\begin{tabular}{llll}
\hline Distribution & KS(p-value $)$ & $\mathrm{W}(\mathrm{p}$-value $)$ & $\mathrm{A}^{2}(\mathrm{p}$-value $)$ \\
\hline TLF & $0.1392(0.6065)$ & $0.5512(0.6939)$ & $0.0897(0.6413)$ \\
FRE2 & $0.1524(0.4892)$ & $0.7597(0.5098)$ & $0.1202(0.4971)$ \\
EP & $0.1164(0.8107)$ & $0.5166(0.7285)$ & $0.0738(0.7320)$ \\
GZ & $0.1149(0.823)$ & $0.6440(0.6060)$ & $0.0836(0.6749)$ \\
LHC & $0.2192(0.1118)$ & $2.176(0.0741)$ & $0.3833(0.0795)$ \\
\hline
\end{tabular}




\section{CONCLUSION}

Some statistical and mathematical properties of the TLF distribution are presented such as the expression for the probability density, and hazard rate functions, survival function, reverse hazard rate function. Further, we have derived the expressions for moments, moment generating function, quantile function, entropies, the skewness, and kurtosis and order statistics measures. The parameters of the proposed distribution are estimated using the MLE method. A real dataset is taken to explore the potentiality of the TLF distribution and concluded that it is more flexible and fits the positive dataset very well than some distributions taken for comparison. We hope that TLF distribution may be a choice in the field of applied statistics, probability theory and reliability analysis.

\section{REFERENCES}

Abbas, S., Taqi, S. A., Mustafa, F., Murtaza, M., \& Shahbaz, M. Q. (2017). Topp-Leone inverse Weibull distribution: theory and application. European Journal of Pure and Applied Mathematics, 10 (5), 1005-1022.

Afify, A. Z., Yousof, H. M., Cordeiro, G. M., Ortega, E. M., \& Nofal, Z. M. (2016). The Weibull Fréchet distribution and its applications. Journal of Applied Statistics, 43(14), 2608-2626.

Afify, A. Z., Hamedani, G., Ghosh, I., \& Mead, M. E. (2014). The transmuted Marshall-Olkin Fréchet distribution: Properties and applications. International Journal of Statistics and Probability.

Al-Shomrani, A., Arif, O., Shawky, A., Hanif, S., \& Shahbaz, M. Q. (2016). Topp-Leone family of distributions: Some properties and application. Pakistan Journal of Statistics and Operation Research, 443-451.

Chaudhary, A. K. \& Kumar, V. (2020). Lindley Half Cauchy distribution: Properties and applications. International Journal for Research in Applied Science \& Engineering Technology (IJRASET), 8 (9), 1233-1242.

da Silva, R. V., de Andrade, T. A., Maciel, D. B., Campos, R. P., \& Cordeiro, G. M. (2013). A new lifetime model: The gamma extended Fréchet distribution. Journal of Statistical Theory and Applications, 12 (1), 39-54.

David, H. A. (1981). Order Statistics, 2nd ed. John Wiley \& Sons

Harlow, D. G. (2002). Applications of the Frechet distribution function. International Journal of Materials and Product Technology, 17(5-6), 482-495. 
Hinkley, D. (1977). On quick choice of power transformation. Journal of the Royal Statistical Society: Series C (Applied Statistics), 26(1), 67-69.

Korkmaz, M. Ç., Yousof, H. M., \& Ali, M. M. (2017). Some theoretical and computational aspects of the odd Lindley Fréchet distribution. Istatistikçiler Dergisi: Istatistik ve Aktüerya, 10(2), 129-140.

Kotz, S., \& Nadarajah, S. (2000). Extreme value distributions: Theory and applications. World Scientific.

Krishna, E., Jose, K. K., Alice, T., \& Ristić, M. M. (2013). The Marshall-Olkin Fréchet distribution. Communications in Statistics-Theory and Methods, 42(22), 4091-4107.

Mahmoud, M. R., \& Mandouh, R. M. (2013). On the transmuted Fréchet distribution. Journal of Applied Sciences Research, 9(10), 5553-5561.

Mead, M. E., Afify, A. Z., Hamedani, G. G., \& Ghosh, I. (2017). The beta exponential Fréchet distribution with applications. Austrian Journal of Statistics, 46(1), 41-63.

Murthy, D.N.P., Xie, M. \& Jiang, R. (2003). Weibull Models, Wiley, New York.

Oguntunde, P. E., Khaleel, M. A., Ahmed, M. T., \& Okagbue, H. I. (2019). The Gompertz fréchet distribution: properties and applications. Cogent Mathematics \& Statistics, 6(1), 1568662.

Oguntunde, P. E., Khaleel, M. A., Okagbue, H. I., \& Odetunmibi, O. A. (2019). The ToppLeone Lomax (TLLo) distribution with applications to airbone communication transceiver dataset. Wireless Personal Communications, 109(1), 349-360.

Smith, R.M. \& Bain, L.J. (1975). An exponential power life-test distribution, Communications in Statistics, 4, 469-481.

Yousof, H. M., Altun, E., \& Hamedani, G. G. (2018). A new extension of Frechet distribution with regression models, residual analysis and characterizations. Journal of Data Science, 16 (4), 743-770.

Yousof, H. M., Butt, N. S., Alotaibi, R. M., Rezk, H., Alomani, G. A., \& Ibrahim, M. (2019). A new compound Fréchet distribution for modeling breaking stress and strengths data. Pakistan Journal of Statistics and Operation Research, 1017-1035. 